\section{Radiation to control gene expression}

Combined radiation therapy and gene therapy offers a very promising strategy for cancer treatment. One problem with gene therapy involves the inappropriate expression of a gene product which may have adverse effects on normal tissues and negate a therapeutic index. Several strategies have been developed to limit gene expression to tumors. These strategies involve targeted vector delivery, tumor specific promoters, and inducible promoter systems. The targetability of radiation therapy offers a unique opportunity to control therapeutic gene expression within tumors via promoter regions of radiation inducible genes. The first radiation inducible promoter system used in combination with gene therapy involved the Egr-1 promoter with the gene for the radiosensitizing cytokine $\mathrm{TNF} \alpha$ which resulted in increased tumor growth inhibition compared with tumors treated with radiation alone. ${ }^{1,2}$ Other groups have used the Egr-1 promoter with the gene for HSVtk which produced enhanced tumor cell killing in the presence of the prodrug ganciclovir following radiation treatment. ${ }^{3,4}$ Ionizing radiation activates transcription of the Egr-1 gene and the $C A r G\left[C C(A+T \text { rich })_{6} G G\right]$ domain of the promoter which are important for this response. ${ }^{5}$ A synthetic promoter has been described based on four copies of the $C A r G$ element of the Egr-1 promoter serving as the enhancer for the $C M V$ promoter. ${ }^{6}$ Several other genes are also induced by ionizing radiation. ${ }^{7}$ The utility of the promoters of these radiation inducible genes has not been evaluated.

In this issue of Gene Therapy two reports concerning radiation inducible promoter systems are presented. Worthington et $\mathrm{al}^{8}$ used the promoter from the radiation inducible WAF1 gene ${ }^{9}$ to achieve radiation induced expression of a reporter gene (green fluorescent protein, $G F P$ ) and a gene that may improve radiation therapy by altering the tumor microenvironment (the inducible form of nitric oxide synthase, iNOS). The ability of the WAF1 promoter to induce gene expression following radiation was tested in an in vitro model with HMEC-1 human endothelial cells and in an ex vivo rat tail arterial segment model. Irradiation of both HMEC-1 cells and rat tail arterial segments transfected with a WAF1/GFP construct with 4 Gy resulted in a significant increase (9.5- and 4.5fold) in GFP expression compared with controls over time. However, the increase did not follow a doseresponse relationship, since $4 \mathrm{~Gy}$ induced greater expression than $6 \mathrm{~Gy}$ in both systems. In the rat arterial segment model, similar results were observed for induction of iNOS expression. The biological activity of the induced iNOS enzyme, which produces nitric oxide that dilates blood vessels, was tested with plasmid vector WAF1/iNOS transfected and irradiated rat arterial segments following addition of the vasoconstrictive drug phenylephrine. Expression of the $i N O S$ enzyme resulted in a significant relaxation of the rat arterial segments. The levels of gene expression using the WAF1 promoter were equal to or greater than with the previously published radiation inducible promoters. The authors propose that dilation of tumor vasculature would increase oxygenation of tumors to improve radiation therapy response or increase delivery of chemotherapeutic agents. Demonstration of the therapeutic efficacy of this combined modality approach of gene therapy with radiation or chemotherapeutic drugs will be important.

Scott et $a l^{10}$ report the use of a 'molecular switch' employing the cre/loxP recombination system and radiation to achieve enhanced and prolonged expression of a reporter gene (GFP) and a therapeutic gene (HSVtk). This approach requires two plasmids, one containing the cre recombinase with a synthetic radiation responsive enhancer/promoter, and the other with the therapeutic gene of interest with a loxP 'stop cassette' between the promoter and gene which prevents transcription. These investigators confirmed that the cre recombinase lead to expression of GFP with the 'stop cassette' when both plasmids used the $C M V$ promoter. The $C M V$ promoter was exchanged with a synthetic promoter containing the radiation response element $(C A r G)$ of the $E g r-1$ promoter driving expression of cre recombinase. GFP expression with the two plasmid system increased both the number of positive cells by 8.2 -fold and the mean fluorescence intensity 14.4-fold compared with a single plasmid with the synthetic promoter and the GFP gene. The efficacy of the 'molecular switch' approach was confirmed using the HSVtk gene and ganciclovir in combination with radiation in which there was increased MCF-7 breast cancer cell growth inhibition of $27 \%$ following a dose of $2 \mathrm{~Gy}$ compared with $8 \%$ for control irradiated and ganciclovir treated MCF-7 cells with only the synthetic promoter/HSVtk construct. Importantly, the level of killing in the radiation-induced system was equal to that achieved with cells transfected with the HSVtk gene driven by the $C M V$ promoter.

A major limitation of the approach described by Scott et $a l^{10}$ is that high levels of tumor specificity are not achieved. As noted by these investigators the use of tumor specific promoters controlling the therapeutic gene combined with the cre/loxP gene under control of the radiation inducible promoter may improve the specificity. Another method to increase the specificity of gene 
expression may be accomplished by targeted vectors. The approach of Worthington et al is well suited for this strategy. The utility of the administration of endothelial cells as gene therapy vectors to target regions of neovasculature has been described. ${ }^{11} \mathrm{~A}$ strategy could be envisioned where endothelial cells transformed with a vasoactive gene under the control of a radiation inducible promoter or 'molecular switch' could achieve highly specific gene delivery. One advantage of a gene such as iNOS is that a large proportion of cells may not have to be transformed to achieve a significant biological effect due to the diffusion of nitric oxide. This advantage may also apply to HSV tk but to a more limited extent due to the requirement for cell-to-cell communication.

A limitation of many of the radiation inducible promoter systems described to date is that they require a fairly high dose (at least 4 or 5 Gy per fraction) to achieve significant gene expression, although Scott et al did observe increased cytotoxicity at 2 Gy. Scott et al claim that prolonged gene expression can be achieved with their approach. The hypothesis that once cre recombinase is produced it will continue to create a functional therapeutic transcript has not been tested. The durability of expression of the cre gene and the stability of the cre enzyme will be critical determinants for the continued expression of the therapeutic gene.

Another issue relates to the effectiveness of increasing the oxygen level in the tumor combined with radiation therapy. A variety of strategies to reduce hypoxia in tumors have had little impact on the radiation response of tumors. One idea presented by Scott et al relates to inhibiting tumor vasculature by antisense and then treating with bioreductive drugs which have shown clinical efficacy. Recently, inhibition of tumor vasculature with angiostatin resulted in increased efficacy of radiation therapy in mouse models of cancer. ${ }^{12}$ Further tests of these different approaches to achieve enhanced killing of tumor cells may determine which strategy has more clinical promise. It will be necessary to validate these strategies with relevant gene therapy vector systems and tumor models. The two reports in this issue of Gene Therapy highlight important approaches to employ radiation to achieve high levels of specific gene expression which may increase the therapeutic index in cancer therapy.
MA Stackhouse and DJ Buchsbaum Department of Radiation Oncology University of Alabama at Birmingham Wallace Tumor Institute-674 1824 6th Avenue South Birmingham, AL 35294, USA

\section{References}

1 Hallahan DE et al. Spatial and temporal control of gene therapy using ionizing radiation. Nature Med 1995; 1: 786-791.

2 Staba M-J et al. Adenoviral TNF- $\alpha$ gene therapy and radiation damage tumor vasculature in a human malignant glioma xenograft. Gene Therapy 1998; 5: 293-300.

3 Joki T, Nakamura M, Ohno T. Activation of the radiosensitive EGR-1 promoter induces expression of the herpes simplex virus thymidine kinase gene and sensitivity of human glioma cells to ganciclovir. Hum Gene Ther 1995; 6: 1507-1513.

4 Kawashita $Y$ et al. Regression of hepatocellular carcinoma in vitro and in vivo by radiosensitizing suicide gene therapy under the inducible and spatial control of radiation. Hum Gene Ther 1999; 10: 1509-1519.

5 Datta $\mathrm{R}$ et al. Ionizing radiation activates transcription of the EGR1 gene via CArG elements. Proc Natl Acad Sci USA 1992; 89: 10149-10153.

6 Marples B et al. Development of synthetic promoters for radiation-mediated gene therapy. Gene Therapy 2000; 7: 511-517.

7 Maity A, Kao GD, Muschel RJ, McKenna WG. Potential molecular targets for manipulating the radiation response. Int J Radiat Oncol Biol Phys 1997; 37: 639-653.

8 Worthington $\mathrm{J}$ et al. Modification of vascular tone using iNOS under the control of a radiation-inducible promoter. Gene Therapy 2000; 7: 1126-1131.

9 el-Deiry WS et al. WAF1, a potential mediator of p53 tumor suppression. Cell 1993; 75: 817-825.

10 Scott SD et al. A radiation-controlled molecular switch for use in gene therapy. Gene Therapy 2000; 7: 1121-1125.

11 Gómez-Navarro J et al. Genetically modified $\mathrm{CD}^{+} 4^{+}$cells as cellular vehicles for gene delivery into areas of angiogenesis in a rhesus model. Gene Therapy 2000; 7: 43-52.

12 Mauceri $\mathrm{HJ}$ et al. Combined effects of angiostatin and ionizing radiation in antitumour therapy. Nature 1998; 394: 287-291. 